\title{
Multimerization of Staufen1 in live cells
}

\author{
CATHERINE MARTEL, ${ }^{1,2,3}$ SAMUEL DUGRÉ-BRISSON, ${ }^{1}$ KARINE BOULAY, ${ }^{1}$ BILLY BRETON, GABRIEL LAPOINTE, \\ SYLVAIN ARMANDO, VÉRONIQUE TRÉPANIER, THOMAS DUCHAÎNE, ${ }^{4,5}$ MICHEL BOUVIER, \\ and LUC DESGROSEILLERS \\ Département de Biochimie, Université de Montréal, Montréal, Québec H3C 3J7, Canada
}

\begin{abstract}
Transport of mRNA is an efficient mechanism to target proteins to specific regions of a cell. Although it is well documented that mRNAs are transported in ribonucleoprotein (RNP) complexes, several of the mechanisms involved in complex formation and localization are poorly understood. Staufen (Stau) 1, a double-stranded RNA-binding protein, is a well accepted marker of mRNA transport complexes. In this manuscript, we provide evidence that Stau1 self-associates in live cells using immunoprecipitation and bioluminescence resonance energy transfer (BRET) assays. The double-stranded RNA-binding domains dsRBD3 and dsRBD4 contributed about half of the signal, suggesting that Stau1 RNA-binding activity is involved in Stau1 selfassociation. Protein-protein interaction also occurred, via dsRBD5 and dsRBD2, as shown by in vitro pull-down, yeast twohybrid, and BRET assays in live cells. Interestingly, Stau1 self-association contributes to the formation of oligomeric complexes as evidenced by the coexpression of split Renilla luciferase halves covalently linked to Stau1 in a protein complementation assay (PCA) combined with a BRET assay with Stau1-YFP. Moreover, we showed that these higher-order Stau1-containing complexes carry RNAs when the RNA stain SYTO 14 was used as the energy acceptor in the PCA/BRET assay. The oligomeric composition of Stau1-containing complexes and the presence of specific mRNAs have been confirmed by biochemical approaches involving two successive immunoprecipitations of Stau1-tagged molecules followed by qRT-PCR amplification. Altogether, these results indicate that Stau1 self-associates in mRNPs via its multiple functional domains that can select mRNAs to be transported and establish protein-protein interaction.
\end{abstract}

Keywords: Staufen; ribonucleoprotein; mRNA transport; mRNA granule; mRNA particle

\section{INTRODUCTION}

Cytoplasmic mRNA transport and local translation to defined subcellular domains allow efficient spatial and temporal restriction of genetic expression (Sossin and DesGroseillers 2006; Rodriguez et al. 2008; Martin and Ephrussi 2009). This phenomenon is observed in a large variety of cell types and organisms and has been shown to play an important role in processes such as learning and memory, synaptic transmission, axis formation during development, cell motility, and asymmetric cell division. Evidence supports a model in which

\footnotetext{
${ }^{1}$ These authors contributed equally to this work.

Present addresses: ${ }^{2}$ Department of Plant Biology, Cornell University, Ithaca, NY 14853, USA; ${ }^{3}$ Boyce Thompson Institute for Plant Research, Cornell University, Ithaca, NY 14853, USA; ${ }^{4}$ Department of Biochemistry, McGill University, Montréal, Québec H3G 1Y6, Canada; ${ }^{5}$ McGill Cancer Center, McGill University, Montréal, Québec H3G 1Y6, Canada.

Reprint requests to: Luc DesGroseillers, Département de Biochimie, Université de Montréal, PO Box 6128, Centre Ville, Montréal, Québec H3C 3J7, Canada; e-mail: luc.desgroseillers@umontreal.ca; fax: (514) 343-2210.

Article published online ahead of print. Article and publication date are at http://www.rnajournal.org/cgi/doi/10.1261/rna.1664210.
}

the differential delivery of new mRNAs to subcellular domains occurs in motile ribonucleoprotein (RNP) structures. A compelling framework was suggested about how mRNA localization could be achieved in an ordered, multi-step pathway. It predicted (1) the formation of mRNPs as a functional complex, (2) the transport of these particles to their destination via cytoskeletal elements and motor proteins, and (3) the derepression of translation of the localized mRNAs. RNA-binding proteins (RBP) become associated with mRNAs either in the nucleus or during remodeling of the complex in the cytoplasm, forming a heterogeneous population of transport complexes. While the composition of mRNA transport complexes begins to be elucidated, less is known about the role(s) of these various RBPs in the mechanism of transport.

Staufen is a member of the double-stranded RNA-binding protein (dsRBP) family. It was first described in Drosophila melanogaster where it is required during development for the localization of the oskar and bicoid transcripts at the posterior and anterior poles of the oocytes, respectively ( $\mathrm{St}$ Johnston et al. 1991). Staufen was also shown to be required for translational derepression of osk mRNA once properly 
localized (Kim-Ha et al. 1995; Micklem et al. 2000). In mammals, two homologous Staufen genes, Stau1 and Stau2, were identified (DesGroseillers and Lemieux 1996; Buchner et al. 1999; Kiebler et al. 1999; Marion et al. 1999; Wickham et al. 1999; Duchaine et al. 2002). Staul is a multifunctional protein involved in different pathways of RNA metabolism. In neurons, Staul is required for the transport and localization of specific mRNAs to dendrites (Kiebler et al. 1999; Kanai et al. 2004; Vessey et al. 2008). Disruption of Staufen blocks transcription-dependent forms of synaptic plasticity/ memory in Drosophila (Dubnau et al. 2003), Aplysia (Liu et al. 2006), and rodents (Lebeau et al. 2008). In addition to its role in mRNA transport, Staul plays additional roles in the regulation of post-transcriptional mRNA expression. When bound to the $3^{\prime}$ UTR of mRNAs, Staul recruits the nonsense mediated decay (NMD) factor Upf1 and triggers degradation of the bound mRNAs (Kim et al. 2005, 2007). In contrast to the NMD pathway that recognizes and degrades mRNAs with a premature termination codon, Stau1mediated mRNA decay is not a quality control mechanism but rather a bona fide mechanism of regulation of gene expression modulating the stability of natural mRNAs. In contrast, when bound to the 5' UTR of mRNAs, Staul enhances their translational efficiency (Dugre-Brisson et al. 2005).

Staul is a modular protein characterized by the presence of two conserved consensus amino acid sequences that fold into a domain involved in double-stranded RNA binding (dsRBD3, dsRBD4) (Marion et al. 1999; Wickham et al. 1999). The $\alpha-\beta-\beta-\beta-\alpha$ structure of this domain interacts with the phosphate backbone of the double helix RNA in its minor and major grooves (Ryter and Schultz 1998; Ramos et al. 2000). Staul also contains two truncated dsRBDs (dsRBD2, dsRBD5) in which amino acid sequence conservation is restricted to the C-terminal half of the consensus (Lunde et al. 2007). These domains are unable to bind RNA. A tubulin-binding domain (TBD) is located between dsRBD4 and dsRBD5 and is believed to be involved in mRNA transport on the cytoskeleton (Wickham et al. 1999). Proteomic and cell biological experiments have clearly established that Staul is a component of ribonucleoprotein complexes in several cell types (Krichevsky and Kosik 2001; Mallardo et al. 2003; Brendel et al. 2004; Villace et al. 2004).

Although it is well accepted that Staul is a marker of mRNA transport complexes and a crucial player for mRNA transport (Kanai et al. 2004; Sossin and DesGroseillers 2006; Vessey et al. 2008), its exact roles within RNPs are still unclear. It is known that several proteins of the dsRBP family such as TRBP2, XlrbpA, ADAR2, PACT, and Rnt1p have the capacity to dimerize through their dsRBDs (Daher et al. 2001; Peters et al. 2001; Valente and Nishikura 2007). For many of these proteins, structural rearrangements resulting from homo- and/or heterodimerization are required for functional activation, and, therefore, dimerization may serve as a molecular regulator. Therefore, we tested whether Stau1, in addition to its other roles in mRNA processing, organizes Staul-containing mRNPs through self-multimerization. In this manuscript, we provide evidence that Staul self-interacts in live cells to form multimeric structures and identify the molecular determinants involved in this property. We further show that these complexes contain specific RNAs.

\section{RESULTS}

\section{Differentially tagged Stau1 are found in common complexes in vivo}

To determine whether Staul forms complexes in mammalian cells, we used immunoprecipitation of differentially tagged Stau1 proteins. HEK293T cells were co-transfected with plasmids coding for Stau1- $\mathrm{HA}_{3}$ and either poly(A)binding protein yellow fluorescent protein (PABP-YFP), Stau1-YFP, or YFP. Western blot experiments indicated that transfected proteins were well expressed and that the levels of expression of Stau1-HA 3 and Stau1-YFP were close to that of endogenous Stau1 (Fig. 1A). Proteins in the cell extracts were then immunoprecipitated using anti-GFP antibody, and co-immunoprecipitated proteins were analyzed by Western blotting (Fig. 1A). In these conditions, Staul$\mathrm{HA}_{3}$ was co-immunoprecipitated with Stau1-YFP and to a lesser extent with PABP-YFP. In contrast, Stau1-HA 3 was not co-immunoprecipitated when co-expressed with YFP. Interestingly, endogenous Stau $1^{55}$ was also found in the precipitates, revealing the biological relevance of these interactions. Treatment of the cell extracts with RNase A before immunoprecipitation did not abolish Stau1/Staul interactions but prevented PABP-YFP/Stau1-HA 3 interaction. These results show that endogenous and differentially tagged Staul are found in common complexes and that, if they are bound to RNA, their association is close enough to be protected from RNase degradation, in contrast to that of Stau1-PABP.

To determine if Staul monomers can self-associate in these complexes, we used an in vitro pull-down assay. Bacterially expressed and purified his 6 -Staul and his ${ }_{6}$-NEP, used as negative controls, were loaded on Ni-NTA columns. In vitro synthesized ${ }^{35}$ S-labeled Staul was loaded on the his $6^{-}$ Staul and his ${ }_{6}$-NEP columns. After several washing steps, his $_{6}$-Staul and his ${ }_{6}$-NEP were eluted from the columns, and the co-eluted ${ }^{35}$ S-labeled Staul was detected by SDS-PAGE and autoradiography (Fig. 1B). Whereas ${ }^{35}$ S-labeled Stau1 was retained on the his $_{6}$-Staul column, it was absent from the his ${ }_{6}$-NEP column, showing that Staul interacts with itself in this assay. The Staul-Staul interaction experiment was done in the presence of RNase A $(50 \mu \mathrm{g} / \mathrm{mL})$. In these conditions, ${ }^{35}$ S-labeled Staul still interacted with his ${ }_{6}$-Staul. These results indicate that Staul has the capacity to dimerize if not oligomerize and that protein-protein interactions contribute at least in part to Staul self-interaction. 
A
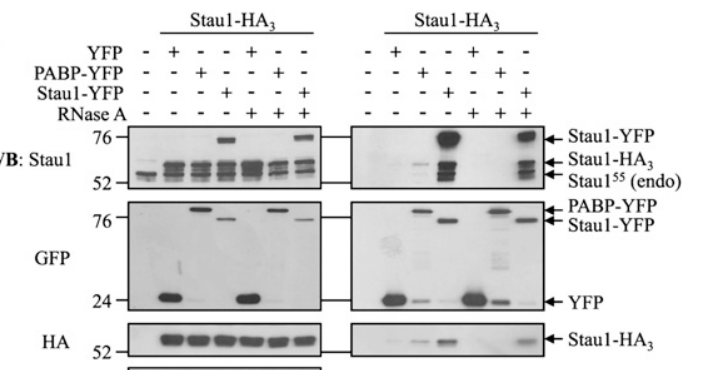

$\beta$-Actin 38 -20-C

Ethidium $=ニ ニ= \pm 28 \mathrm{~S}$ rRNA

Ethidium
bromide

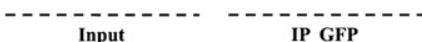

B

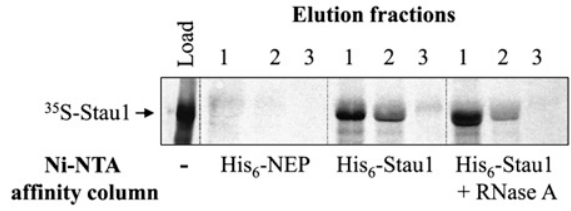

FIGURE 1. Differentially tagged-Stauls are found in common complexes. (A) HEK293T cells were mock transfected or co-transfected with Staul-HA 3 expressor and either YFP, PABP-YFP, or Staul-YFP expressors in the absence or presence of RNase A as indicated. An aliquot of the proteins in the cell extracts was separated by SDS-PAGE (left panels, "Input") and the remaining extracts were immunoprecipitated using anti-GFP antibody (right panels, "IP GFP"). Proteins were analyzed by Western blotting using anti-Staul, anti-GFP, and anti-HA antibodies, as indicated. Anti- $\beta$-actin antibody was used as a loading control. Molecular weight markers are indicated on the left side of the gels. As control for RNase A digestion, total RNAs were isolated and separated on agarose gel. Ribosomal RNAs (rRNA) were detected by ethidium bromide staining. (B) Bacterially expressed his ${ }_{6}$-Staul or his ${ }_{6}$ NEP (as control) fusion proteins were fixed on a Ni-NTA column and extensively washed. In vitro synthesized ${ }^{35} \mathrm{~S}$-Staul was loaded onto the columns. Columns were washed and then his ${ }_{6}$-Staul and his 6 -NEP were eluted and collected in three successively fractions (lanes 1-3). The co-eluted ${ }^{35} \mathrm{~S}$-Staul protein was separated by SDS-PAGE and visualized by autoradiography. In parallel, his $_{6}$-Staul and ${ }^{35}$ S-Staul preparations were treated with RNase A before loading onto the columns. An aliquot of ${ }^{35}$ S-Staul was also loaded on the gel (lane, "Load") to control for its synthesis and size.

\section{Stau1 self-associates in live cells}

Our results indicate that several Staul monomers are found in common complexes in vivo and that direct interaction may contribute to complex formation. In order to confirm the proximity (or interaction) of Staul molecules within mRNPs in live cells, bioluminescence resonance energy transfer (BRET) assays were used (Fig. 2A). In this technique, one candidate interacting protein is fused to Renilla luciferase, a luminescent energy donor, and the other to YFP, a fluorescent energy acceptor. The two fusion proteins are then co-expressed in the same cells. If the two proteins interact, their close proximity allows nonradiative energy transfer (BRET) between the luciferase and the YFP. BRET does not occur if the two proteins are separated by $>100 \AA$, making the technique ideal for monitoring protein-protein interactions in biological systems. Therefore, Rluc and YFP were fused at the $\mathrm{C}$ terminus of Staul. Fixed amounts of Staul-Rluc were transfected in combination with increasing amounts of Stau1-YFP (Fig. 2B), PABP-YFP, or YFP to generate BRET saturation curves (Fig. 2C). Co-expression of Staul-Rluc and Stau1-YFP produced a strong BRET signal

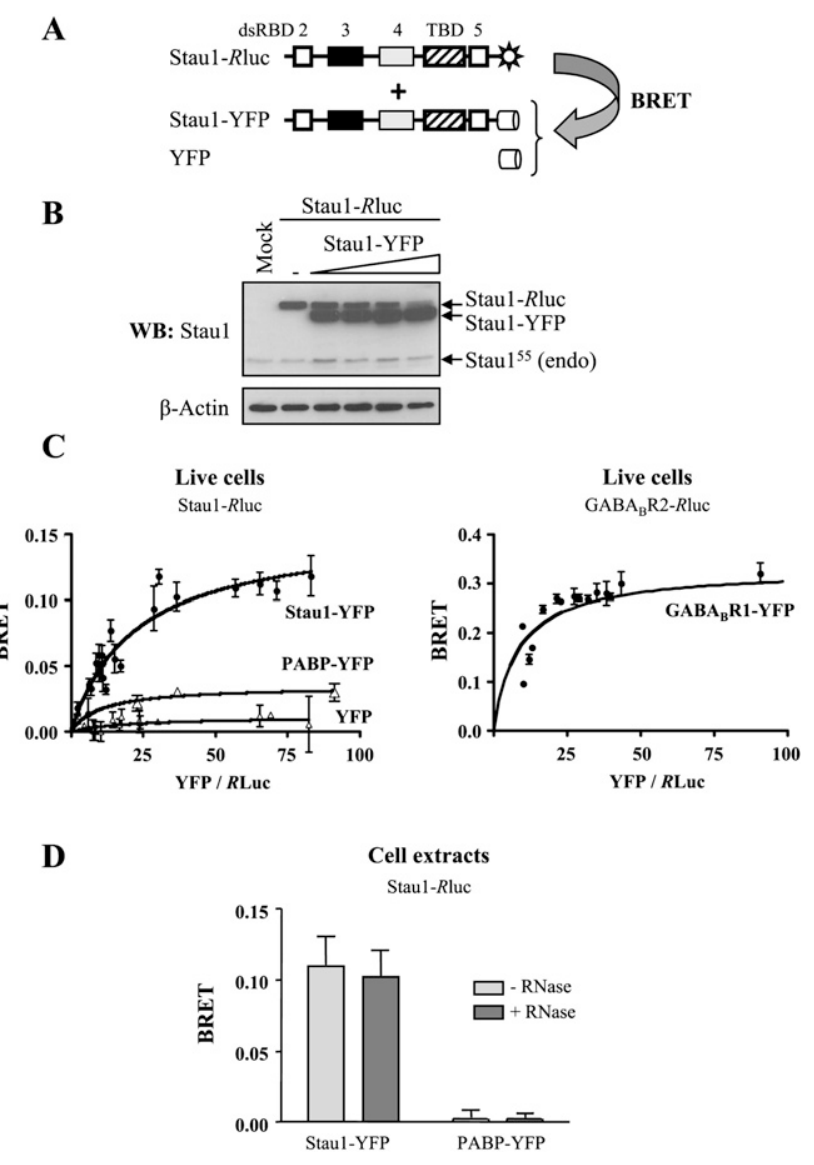

FIGURE 2. Staul self-associates in live cells as measured by BRET. (A) Schematic representation of the BRET assay. (Black and gray boxes) Double-stranded RNA-binding domains with major (dsRBD3) and minor (dsRBD4) dsRNA-binding activity, respectively; (white boxes) domains with no demonstrated RNA-binding activity (dsRBD2 and dsRBD5); (hatched boxes) tubulin-binding domain (TBD). (Sun) Rluc, (cylinder) YFP. $(B, C)$ Plasmids coding for Staul-Rluc were cotransfected in HEK293T cells with various amounts of Stau1-YFP, PABP, or YFP expressors. (B) Expression of Stau1-Rluc and Stau1-YFP proteins using Western blotting with anti-Staul antibody. $\beta$-actin antibody was used as a loading control. (C) BRET saturation curves were generated to provide evidence of specific interactions between the proteins in live cells. BRET ratios were defined as described in the Materials and Methods and plotted as a function of the excited YFP activity to total Renilla luciferase activity ratio. (Left) Comparison of BRET saturation curves when Staul-Rluc was co-expressed with Stau1YFP, PABP-YFP, or YFP. Error bars correspond to standard deviation. (Right) $\mathrm{GABA}_{\mathrm{B}}$ receptor 2-Rluc and $\mathrm{GABA}_{\mathrm{B}}$ receptor 1-YFP, two proteins known to form strong functional dimer, were used as a BRETpositive control. (D) HEK293T cells were co-transfected with Stau1Rluc and either Staul-YFP or PABP-YFP expressors as described in $B$. Cells were lysed and cell extracts were incubated in the presence or absence of RNase A for $15 \mathrm{~min}$ at room temperature. BRET ratios at saturation in the presence $(+)$ or absence $(-)$ of RNase are shown. $n=3$. Error bars correspond to standard deviation. 
in live cells comparable to that of proteins known to form obligatory dimers (Fig. $2 \mathrm{C}, \mathrm{GABA}_{\mathrm{B}}$ receptors), suggesting a close proximity between these two proteins. In contrast, Staul-Rluc only produced a negligible BRET signal when coexpressed with PABP-YFP or YFP, confirming the specificity of the Stau1-Rluc/Stau1-YFP BRET signal and suggesting that the binding of two RNA-binding proteins on RNA molecules is not sufficient to induce a strong BRET signal. The BRET assay was then performed with cell extracts, allowing us to determine whether the interaction is sensitive to RNase treatment or not. Cells were lysed and the resulting cell extracts were incubated in the presence or absence of RNase A for $15 \mathrm{~min}$ at room temperature before fluorescence measurement. There was no significant difference in Staul-Rluc/Staul-YFP BRET signals between RNase A-treated and untreated extracts (Fig. 2D).

\section{RNA-binding activity contributes to Stau 1 self-association in live cells}

Although Staul self-association is resistant to RNase treatment and can occur with purified proteins in vitro, it is possible that an RNA molecule may be required during an initial step that triggers Staul self-association or that a linker RNA is protected from RNase A degradation by bound Staul molecules. To determine whether Staul RNA-binding activity is involved in Staul self-association, RNA-bindingdefective Staul mutants were fused to YFP and used in the BRET assay described above to determine their ability to interact with the wild-type Stau1-Rluc. Mutations were introduced in Staul dsRBD3, a domain shown to carry the major Staul RNA-binding activity, and in dsRBD4, a domain carrying a weak RNA-binding activity in vitro (Fig. 3A; Wickham et al. 1999). Four mutants were generated by either deletion of the complete dsRBD3 (Stau1 ${ }^{\Delta 3}$-YFP) or dsRBD4 (Stau1 ${ }^{\Delta 4}$-YFP) or through point mutations in amino acids required for RNA binding in dsRBD3 (Stau1 ${ }^{\mathrm{KK}}$-YFP) or in both dsRBD3 and dsRBD4 (Stau1 ${ }^{4 \mathrm{~K}_{-}}$ YFP). In these mutants, lysines 153 and 154 in dsRBD3 and lysines 256 and 257 in dsRBD4 were mutated to alanines. HEK293T cells were co-transfected with plasmids coding for Stau1-Rluc and either one of the mutated fusion proteins (Fig. 3B). BRET signals were calculated in live cells $24 \mathrm{~h}$ post-transfection. Following expression of mutants in dsRBD3 alone (Stau1 ${ }^{\mathrm{KK}}$ and Stau1 ${ }^{\Delta 3}$ ), the BRET ratio was weakly affected, being only reduced by $\sim 20 \%(77 \% \pm 10 \%$ and $77.5 \pm 0.9 \%$, respectively) as compared with the BRET activity generated with the wild-type Staul-YFP (Fig. 3C). When mutations were introduced in both dsRBD3 and dsRBD4 (Stau $\left.1^{4 \mathrm{~K}}\right)$, the BRET ratio was further reduced to $51.8 \%( \pm 0.4 \%)$ of the one obtained with Stau1-YFP. These results indicate that Stau1 RNA-binding activity contributes to Staul self-association and may be required to select mRNAs in Stau1-containing mRNPs and/or help to initiate or stabilize protein-protein interaction. Nevertheless, protein-

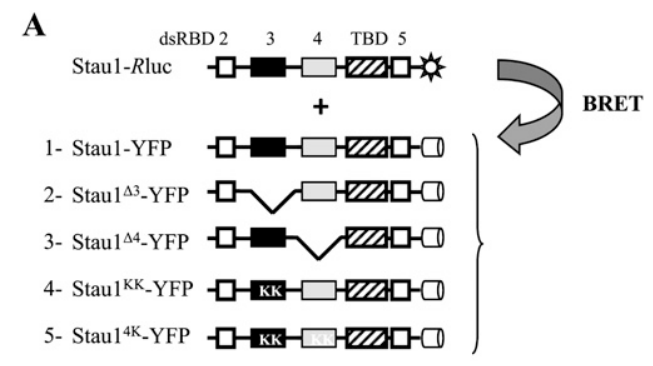

B
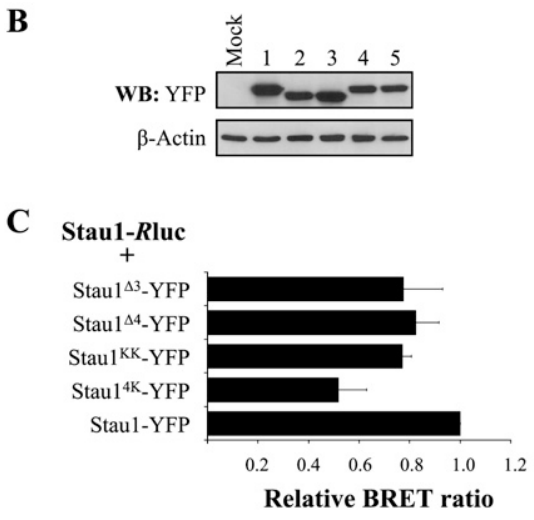

FIGURE 3. Staul RNA-binding activity is involved in complex formation. (A) Schematic representation of Staul mutants. Symbols are described in the legend for Figure 2. (Stau1 ${ }^{\mathrm{KK}}$ ) Mutations K153A and $\mathrm{K} 154 \mathrm{~A}$ in dsRBD3; (Stau ${ }^{4 \mathrm{~K}}$ mutant) mutations $\mathrm{K} 153 \mathrm{~A}$ and K154A in dsRBD3 and K256A and K257A in dsRBD4. (B) Plasmids coding for Staul-YFP and Staul-YFP mutants were transfected in HEK293T cells and protein expression was monitored by Western blotting using anti-GFP antibody. Anti- $\beta$-actin antibody was used as loading control. Numbers above each lane correspond to those in $A$. (C) HEK293T cells were co-transfected with Staul-Rluc and either Staul-YFP, Stau1 ${ }^{\Delta 3}$-YFP, Staul ${ }^{\Delta 4}$-YFP, Stau ${ }^{4 \mathrm{~K}}{ }_{-}$YFP, or Stau $1^{\mathrm{KK}}$-YFP expressors. BRET ratios of wild-type Staul and RNA-binding defective Staul mutants were calculated as in Figure 2, and relative BRET ratios were generated with the Staul-Rluc/Stau1-YFP BRET ratio arbitrarily fixed to $1 . n=3$. Error bars correspond to standard deviation.

protein interaction seems to also contribute to Staul selfassociation in live cells.

\section{Characterization of the protein-protein determinants required for Stau1 self-association}

To identify the molecular determinants involved in protein interaction, we first expressed different combinations of wildtype and Staul deletion mutants (Fig. 4A,B) fused to either Rluc or YFP and measured their BRET activity in live cells (Fig. 4C-E). When co-expressed with wild-type Staul, Stau $^{\Delta 2}$ or Staul ${ }^{\Delta 5}$ expression reduced the BRET activity by $\sim 20 \%$ and $50 \%$, respectively, as compared with that obtained with wild-type Staul (Fig. 4C). These results suggest that dsRBD5 and dsRBD2 are major and minor determinants involved in the protein-protein aspect of Staul self-association, respectively. To further characterize these interactions, we then calculated BRET activity following co-expression of these two fusion proteins: Stau $1^{\Delta 5}$-Rluc/Stau1 ${ }^{\Delta 2}$-YFP, 
A

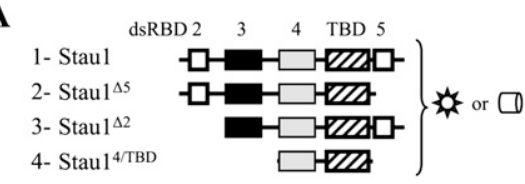

B

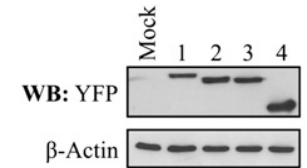

C

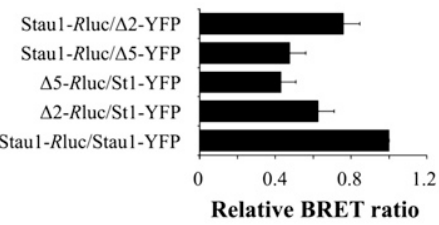

D

$\Delta 2-R$ luc/ $\Delta 2$-YFP

$\Delta 5$-Rluc/ $\Delta 5$-YFP

$\Delta 5$-Rluc/ $\Delta 2$-YFP

$\Delta 2-$ Rluc/ $\Delta 5$-YFP

Stau1-Rluc/Stau1-YFP

$\mathbf{E}$

Staul-Rluc/4TBD-YFP

4TBD-Rluc/Staul-YFP

Staul-Rluc/Staul-YFP

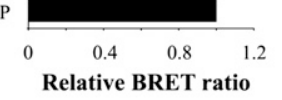

F

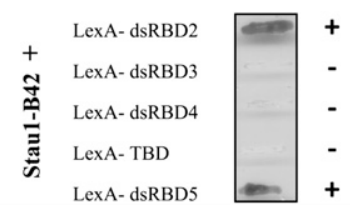

FIGURE 4. Characterization of Staul protein-protein interaction in live cells. (A) Schematic representation of Staul and mutants. Symbols are described in the legend of Figure 2. (B) Plasmids coding for Stau1-YFP and Stau1-YFP mutants were transfected in HEK293T cells and protein expression was monitored by Western blotting using anti-GFP antibody. Anti- $\beta$-actin antibody was used as loading control. Numbers above each lane correspond to those in $A$. $(C-E)$ Cells were co-transfected with different combinations of Staul and Staul deletion mutants fused to either Rluc or YFP as indicated. BRET ratios were calculated as in Figure 2, and relative BRET ratios were generated with the Stau1-Rluc/Staul-YFP BRET ratio arbitrarily fixed to $1 . n=3$. Error bars correspond to standard deviation. $(F)$ Yeast cells were co-transformed with a plasmid coding for Stau1-B42 and different expressors of Staul domains fused to LexA, as indicated. Cells were screened for growth on selective medium and $\beta$-galactosidase expression. Stau1-B42 interacts $(+)$ with Staul dsRBD2 and dsRBD5 but not $(-)$ with dsRBD3, dsRBD4, or TBD fused to LexA in a two-hybrid assay.

Stau $1^{\Delta 2}-R$ luc/Stau $1^{\Delta 2}$-YFP, Stau $1^{\Delta 5}-R$ luc/Stau $1^{\Delta 5}$-YFP, and Stau $1^{\Delta 2}-R$ luc/Stau $1^{\Delta 5}$-YFP. As shown in Figure $4 \mathrm{D}$, coexpression of $\mathrm{Stau}^{\Delta 5}$-Rluc and Stau $1^{\Delta 5}-\mathrm{YFP}$ showed a BRET activity that was reduced to $51.3 \%$ ( $\pm 0.1 \%$ ), consistent with a significant role of dsRBD5 in Staul association. In contrast, co-expression of Stau $1^{\Delta 2}$-Rluc and Stau $1^{\Delta 2}$-YFP generated a BRET signal that was similar to that obtained with Staul-Rluc and Stau1-YFP. Finally, BRET signals generated by the co-expression of the Stau $1^{\Delta 5}$ Rluc/Stau1 ${ }^{\Delta 2}$-YFP or Stau1 ${ }^{\Delta 2}$-Rluc/Stau1 ${ }^{\Delta 5}$-YFP pairs were highly reduced, being only $\sim 25 \%(32.8 \% \pm 0.2 \%$ and $24.3 \% \pm 0.4 \%$, respectively) of the BRET activity generated by the Staul wild-type pair (Fig. 4D). Consistently, a Stau1 mutant that carried only the dsRBD4 and tubulin-binding domains (RBD4/TBD) did not generate BRET signal when expressed with wild-type Staul (Fig. 4E). Altogether, these results indicate that dsRBD5 is clearly involved in Stau1 selfinteraction in live cells, whereas the role of dsRBD2 is less clear.

To confirm that dsRBD5 and dsRBD2 are the molecular determinants involved in Stau1-Staul protein interaction, the yeast two-hybrid system was used. The full-length Stau1 protein was fused to the B42 transcription activator, whereas each of the four dsRBDs of Staul and the tubulin-binding domain (TBD) were individually fused to the LexA DNAbinding domain. After selection for the presence of both plasmids in the yeast strains, cells were plated on a $\mathrm{Ura}^{-}$, $\mathrm{His}^{-}, \mathrm{Trp}^{-}$, $\mathrm{Leu}^{-}$medium with galactose and X-Gal. Our results showed that only yeast cells expressing the dsRBD2and dsRBD5-LexA fusion proteins grew on the selective medium and produced $\beta$-galactosidase activity (Fig. 4F). Therefore, both dsRBD2 and dsRBD5 can interact with the full-length Staul protein in this assay.

\section{Subcellular distribution of Stau1 and Stau1 mutants}

To determine whether the differential BRET responses obtained with the Staul mutants may be explained by major defects in the subcellular distribution of these proteins, Stau1-YFP and Stau1-YFP mutants were expressed in COS-7 cells and their subcellular distribution was observed by fluorescence microscopy. HEK293T cells were not used in this assay because of the small size of their cytoplasm. As previously shown (Marion et al. 1999; Wickham et al. 1999), Stau1-YFP showed a granular distribution in the cytoplasm and nuclear exclusion. Stau $1^{\Delta 5}$-YFP, a mutant that produced weak BRET signals (Fig. 4), had a subcellular distribution similar to that of Staul-YFP (Fig. 5). As compared with Stau1-YFP, Stau $1^{\mathrm{KK}}$-YFP showed an additional weak nucleolar distribution, and mutants in the RNA-binding domains dsRBD3 and dsRBD4 showed cytosolic granules that are slightly bigger than those observed with Staul-YFP. However, this cannot explain the difference in the BRET response of Stau $1^{4 \mathrm{~K}}$-YFP as compared with the others (Fig. 3C). The distribution of 4/TBD-YFP was random and similar to that of YFP alone as previously described (Luo et al. 2002).

\section{Stau1 forms oligomers in live cells}

Knowing that Staul is present in large mRNP complexes, we assessed the capacity of Staul to form higher order 


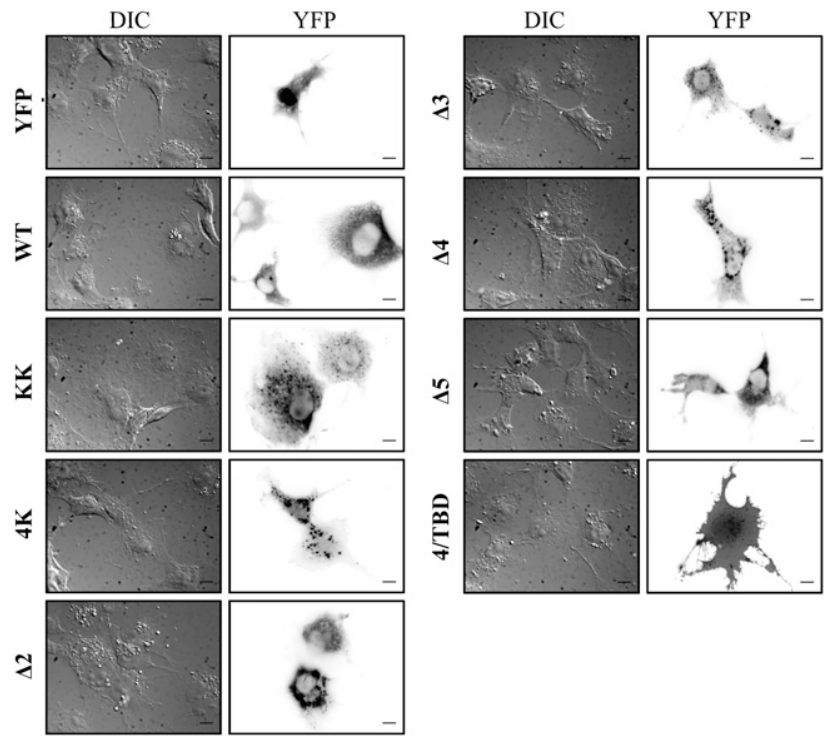

FIGURE 5. Subcellular distribution of Staul and Staul mutants. COS-7 cells were transfected with plasmids coding for Stau1-YFP or Staul-YFP mutants as indicated. YFP expression was observed $24 \mathrm{~h}$ later by fluorescence microscopy. Both differential interference contrast microscopy (DIC) and the YFP constructs are shown.

structures in live cells. To this end, we combined protein complementation (PCA) and BRET assays (Fig. 6A). For the PCA assay, Rluc is cut into two nonfunctional halves $\left(R l u c{ }^{\mathrm{F} 1}\right.$ and $R \operatorname{luc}^{\mathrm{F} 2}$ ). Each half of the split luciferase protein cannot by itself generate light when expressed in cells. However, their fusion to proteins that interact via protein-protein interactions allows the reconstitution of a functional luciferase and light emission. Therefore, the N-terminal half $\left(\right.$ Stau1-Rluc $\left.{ }^{\mathrm{F} 1}\right)$ and the C-terminal half (Stau1-Rluc ${ }^{\mathrm{F} 2}$ ) of a split Rluc protein were fused at the $\mathrm{C}$ terminus of Staul. Consistent with the BRET assay, co-expression of StaulRluc $^{\mathrm{F} 1}$ and Stau1-Rluc ${ }^{\mathrm{F} 2}$ reconstituted a functional luciferase that produced light $(8293 \pm 1341$ arbitrary units [AU]) via Staul-Staul interaction, whereas expression of either fusion protein alone did not $(580 \pm 87$ and $529 \pm 41 \mathrm{AU}$, respectively). Then, to determine whether additional Stau1 molecules can interact with this complex in live cells, the PCA assay with Stau1-Rluc ${ }^{\mathrm{F} 1}$ and Stau1-Rluc ${ }^{\mathrm{F} 2}$ was repeated in the presence of an increasing concentration of Stau1-YFP for BRET measurement. If Staul induces the formation of large complexes, a complete Stau1-Stau1-Rluc will be reconstituted and will transfer energy to the Staul-YFP that will be present in the same complex. As shown in Figure 6B, co-expression of Stau1-Rluc ${ }^{\mathrm{F} 1}$, Stau1-Rluc ${ }^{\mathrm{F} 2}$, and Stau1-YFP produced a strong BRET saturation curve, suggesting that at least three Staul molecules associate in these complexes. These interactions between Staul molecules were resistant to RNase treatment (Fig. 6C), consistent with results shown in Figure 2D. In contrast, Stau1-Rluc ${ }^{\mathrm{F} 1}$ and Stau1-Rluc ${ }^{\mathrm{F} 2}$ did not produce BRET activity when co-expressed with YFP or PABP-YFP (Fig. 6B). Other controls were done with CXCR4, a protein that does not interact with Stau1. Stau1-Rluc ${ }^{\mathrm{F} 1}$ and CXCR4-Rluc ${ }^{\mathrm{F} 2}$ as well as Stau1-Rluc ${ }^{\mathrm{F} 2}$ and CXCR4-Rluc ${ }^{\mathrm{F} 1}$ did not produce significant luciferase activity when coexpressed (533 \pm 59 and $613 \pm 19$ AU, respectively); CXCR4-Rluc ${ }^{\mathrm{F} 1}$ and CXCR4-Rluc ${ }^{\mathrm{F} 2}$, two proteins known to interact in live cells (Hamdan et al. 2006), did not transfer energy to Staul-YFP in the BRET assay (data not shown).

\section{Stau1-containing oligomeric complexes contain RNAs}

To determine whether Staul-containing oligomeric complexes contain RNAs, we modified the live cells assay that combined PCA and BRET to detect RNA-protein interaction (Fig. 7A). In this assay, Stau1-Rluc ${ }^{\mathrm{F} 1}$ and Stau1-Rluc ${ }^{\mathrm{F} 2}$ were co-expressed to reconstitute luciferase activity while SYTO 14, a dye that stains RNA, was used as the energy acceptor. If Staul-containing oligomers associate with RNA, BRET signal will be generated as a consequence of the close proximity between the reconstituted Rluc and SYTO 14. As shown in Figure 7B, expression of both Stau1-Rluc and the split Stau1-Rluc ${ }^{\mathrm{F} 1}$ and Stau1-Rluc ${ }^{\mathrm{F} 2}$ generated BRET signals when RNA was stained with SYTO 14, indicating that RNAs are present in the Staul-containing complex. In contrast, Rluc activity resulting from interaction of the split protein kinase A (PKA) regulatory subunit- $R \mathrm{luc}^{\mathrm{F} 1}$ and PKA catalytic subunit-Rluc ${ }^{\mathrm{F} 2}$ did not induce BRET signal in the presence of SYTO 14. Whereas luciferase activity generated by interaction of the split Rluc fusion proteins was not impaired by RNase treatment (data not shown; Fig. 6C), the observed Staul-Staul-Rluc/SYTO 14 BRET signal was sensitive to RNase treatment, indicating that Staul-containing complexes indeed contain RNAs (Fig. 7C).

To confirm the formation of RNA-containing Stau1oligomers, a biochemical approach involving two successive immunoprecipitation steps was used. HEK293T cells were transfected with plasmids coding for Staul- $\mathrm{HA}_{3}$ and either YFP, Stau1-YFP, and/or Stau1-Flag (Fig. 8A). The level of expression of these proteins was evaluated by Western blotting. First, Staul-containing complexes were immunoprecipitated with anti-Flag antibodies and eluted with the Flag peptide. The resulting complexes contained Staul- $-\mathrm{HA}_{3}$ and Staul-YFP when co-expressed. Then, Stau1-Flag-containing complexes were re-immunoprecipitated using anti-GFP antibody. The resulting pellet also contained Staul- $\mathrm{HA}_{3}$ and Staul-Flag, indicating that the three tagged-Staul proteins were present in the same complexes. As controls, other combinations of transfected Staul-tagged molecules lacking either Stau1-Flag or Stau1-GFP did not bring down Stau1- $\mathrm{HA}_{3}$.

To determine whether the Staul-containing oligomeric complexes contain RNAs, immunoprecipitates obtained from the successive-IPs were treated with TRIzol reagent to isolate putative RNAs, and the resulting material was analyzed by qRT-PCR. Arf1 and PAICS mRNAs, two known Staul-bound mRNAs (Kim et al. 2005), were specifically amplified from samples of the two successive IPs and were 
A
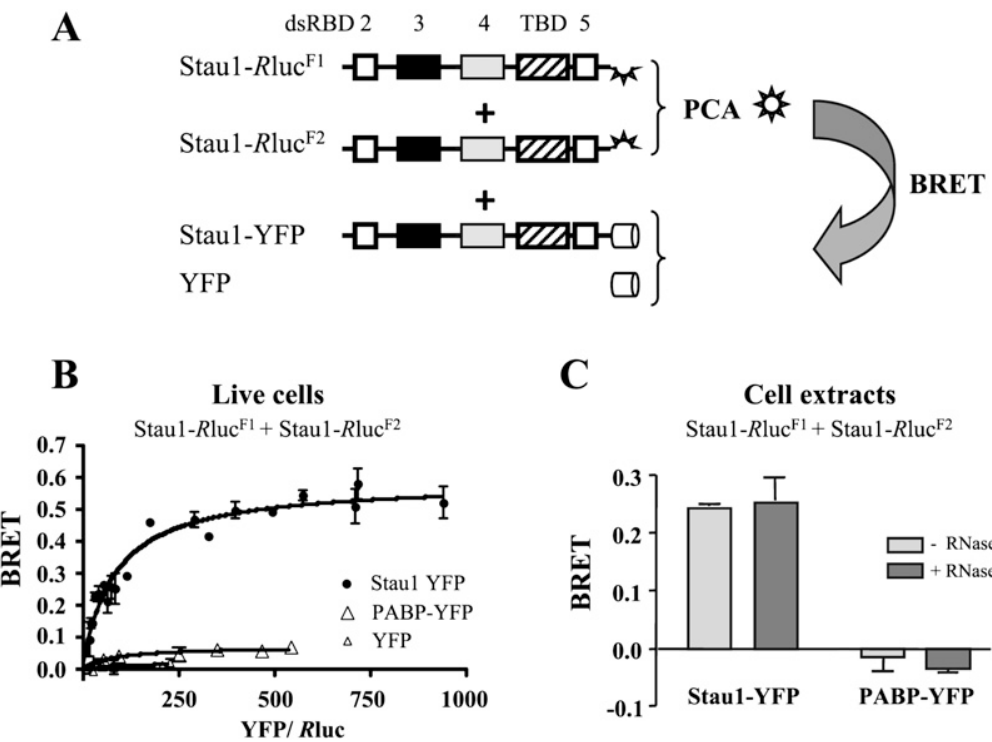

C

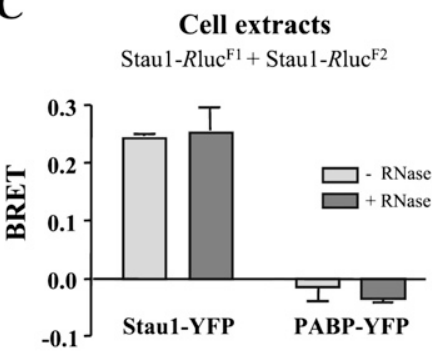

FIGURE 6. Staul forms oligomers in live cells. (A) Schematic representation of the BRET-PCA assay. Staul was fused to either the $\mathrm{N}$-terminal $\left(\operatorname{Rluc}^{\mathrm{F}}\right)$ or the $\mathrm{C}$-terminal $\left(\operatorname{Rluc}^{\mathrm{F} 2}\right)$ of Rluc. Formation of Staul dimers will bring together the two Rluc halves through protein complementation (PCA) and reconstitute a functional luciferase. This enzyme will transfer energy and generate BRET signal if Staul-YFP is also present in close proximity, suggesting the formation of oligomers. (B) HEK293T cells were co-transfected with constant amounts of plasmids coding for Staul-Rluc ${ }^{\mathrm{F} 1}$ and Stau1-Rluc ${ }^{\mathrm{F} 2}$ and increasing amounts of plasmids coding for either Stau1-YFP, PABP-YFP, or YFP. The saturation curves provide evidence of specific interactions between at least three Staul proteins in live cells. BRET ratios were calculated as in Figure 2. Error bars correspond to standard deviation. $(C)$ Plasmids were transfected as in $B$. Cells were lysed and cell extracts were treated with RNase (+RNase) or the buffer alone ( - RNase) before BRET calculation.

absent from other control IPs (Fig. 8B). To discriminate between specific Staul-mRNA association and nonphysiological aggregates of mRNPs, we calculated the ratio between the amount of Arf1 and PAICS mRNAs in the successive immunoprecipitates and their amount in the cells before IPs (IP/input). As a control for specificity, we also calculated the ratio of an abundant non-Staul-interacting mRNA coding for the ribosomal protein RPL22. When the ratio obtained for RPL22 mRNA was arbitrarily normalized to 1 , an enrichment ranging from 13- to 20-fold was observed for Arf1 mRNA and from 32- to 43-fold for PAICS mRNA. Altogether, these results indicate that Arf1 and PAICS mRNAs are highly enriched in the multimeric complexes as compared with non-Staul-interacting controls.

\section{DISCUSSION}

Many proteins self-associate to generate dimers and/or oligomers, and this structural reorganization is essential to control their function, structure, and/or regulation (Marianayagam et al. 2004). Enzymatic regulation, cell signaling, DNA binding, and regulation of gene expression are a few examples among all the molecular activities that are regulated by protein self-interaction. Especially, proteins of the dsRBP family such as PKR, TRBP2, XlrbpA, ADAR2, PACT, and Rntlp are known to form homo- and/or heterodimers (Lamontagne et al. 2000; Daher et al. 2001; Peters et al. 2001; Hitti et al. 2004; Lemaire et al. 2005; Valente and Nishikura 2007). The structural rearrangement resulting from dimerization is required for their functional activation, and therefore dimerization serves as a molecular regulator (Daher et al. 2001; Peters et al. 2001; Valente and Nishikura 2007). In this study, we show that Staul, like other members of the dsRBP family, selfassociates. Using novel approaches in live cells, we further showed that Staul generates multimeric complexes through both RNA-binding activity and proteinprotein self-interaction and that these complexes contain specific RNAs. Our results suggest that Staul has the potential to act as a scaffolding protein that organizes mRNAs into functional mRNPs.

\section{Molecular determinants for mRNP formation}

Our BRET assays indicate that Staul RNA-binding activity or intact dsRBD3 and dsRBD4 contributes for $\sim 50 \%$ of the Staul self-association (Fig. 3). Several biochemical studies (Nanduri et al. 2000) as well as NMR and crystallographic structures of bacterially expressed dsRBDs (Bycroft et al. 1995; Kharrat et al. 1995; Ryter and Schultz 1998; Nanduri et al. 2000; Ramos et al. 2000) indicate that RNA-binding competent dsRBDs behave as monomeric forms. Therefore, it is likely that the contribution of these domains to Staul self-interaction depends on their capability to bind RNA and not on putative protein-protein interaction. In addition, our observation that protein interactions occur via other domains that do not bind RNAs in vitro (Wickham et al. 1999) supports a model in which dsRBD3 and dsRBD4 are free to interact with mRNAs. Staul RNA-binding activity may then be used to recognize and select mRNAs to be included in mRNPs. Subsequently, it may facilitate the association of Stau1 monomers on a dsRNA structure and then facilitate the recognition, formation, and/or maintenance of proteinprotein interaction mediated by dsRBD5 and dsRBD2.

The RNA-binding activity is nevertheless not essential for subsequent protein-protein interaction. Indeed, $\sim 50 \%$ of the BRET activity is still detectable when RNA-binding deficient Staul mutants are expressed in vivo (Fig. 3). Accordingly, Staul self-association can be detected in vitro using purified proteins in the presence of RNase (Fig. 1B), revealing the intrinsic property of Staul to self-associate. 
A

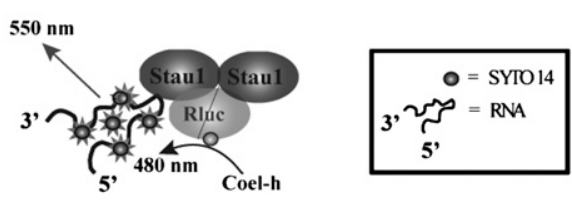

B

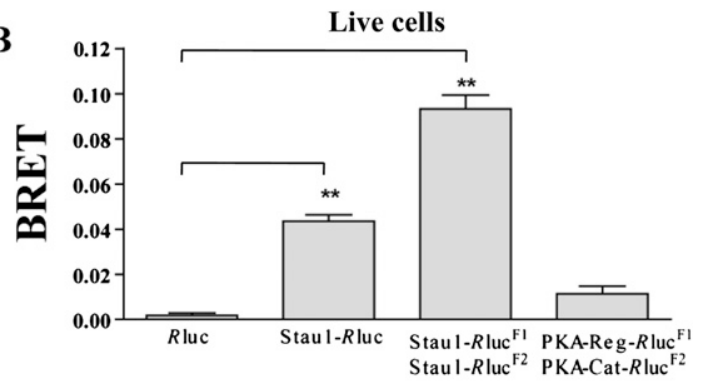

C

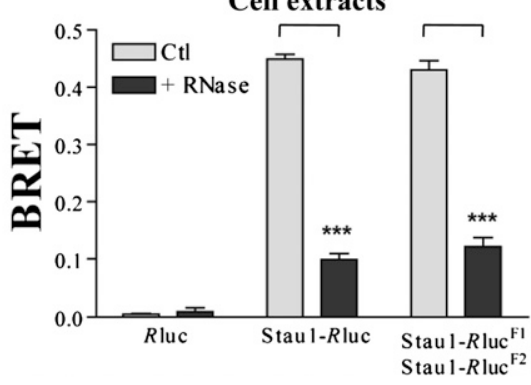

FIGURE 7. Staul oligomers contain RNA in live cells. (A) Schematic representation of the PCA-BRET assay for protein-RNA interaction. As described in Figure 6, Staul-Rluc ${ }^{\mathrm{F} 1}$ and Staul-Rluc ${ }^{\mathrm{F} 2}$ were transfected in HEK293T cells to reconstitute a functional luciferase as a consequence of Staul-Staul interaction. Then cells were treated with SYTO 14, a fluorescent dye that stains RNA. Energy transfer can occur from the reconstituted Rluc to SYTO 14 if in close proximity and generate BRET signal. (B) BRET ratios at saturation are indicated for each association between monomeric (Rluc, Staul-Rluc) or split (Staul$R_{\text {luc }}{ }^{\mathrm{F} 1}+$ Staul-Rluc ${ }^{\mathrm{F} 2}$, PKA reg-Rluc ${ }^{\mathrm{F} 1}+\mathrm{PKA}$ cat-Rluc ${ }^{\mathrm{F} 2}$ ) Rluc and SYTO 14. Error bars are standard deviation. $\left.{ }^{* *}\right) P<0.01$ (ANOVA and post-test Dunnett). (C) Transfected HEK293T cells were lysed and cell extracts were treated with RNase (+RNase) or the buffer alone $(\mathrm{Ctl})$ before adding SYTO 14 for the BRET assay. ( $\left.{ }^{* * *}\right) P<0.001$ ( $t$-test). Note that the BRET values are higher in cell extracts than those in live cells probably due to the better availability of SYTO 14 in cell extracts.

This is consistent with previous studies in which dsRBPs form dimers through both dsRBDs and other domains not involved in RNA binding (Lamontagne et al. 2000; Daher et al. 2001; Peters et al. 2001; Hitti et al. 2004; Valente and Nishikura 2007). Our results identified dsRBD5 as the major determinant for protein-protein interaction, contributing to $\sim 50 \%$ of the Staul self-interaction (Fig. 4C). Homophilic interaction between dsRBD5s may contribute to stabilize particle formation on mRNAs. The role of dsRBD2 is less clear. Although it can interact with Stau1 in the yeast assay (Fig. 4F), its deletion has no impact on the BRET signal in live cells (Fig. 4C). Interestingly, heterophilic interactions between dsRBD5 and the N-terminal end of Staul (in- cluding dsRBD2) were observed in the yeast two-hybrid assay (data not shown). This observation would permit generating large multimeric complexes through homo- and heterophilic interactions between dsRBDs. Moreover, it opens the additional possibility that Staul structure and/or functions may be regulated by intramolecular interaction between the $\mathrm{N}$-terminal domain and dsRBD5. A similar regulatory mechanism has been described for Rnt1p, a member of the dsRBP family in yeast, in which an N-terminal domain established intramolecular interaction with the C-terminal dsRBD (Lamontagne et al. 2000). Competition between intramolecular Nter-dsRBD5 interaction and intermolecular homophilic binding of dsRBD5 may control Staul self-association and, as a consequence, Staul structure/functions. Alternatively, deletion of dsRBD2 may somehow destabilize dsRBD3-mediated RNA-binding activity.
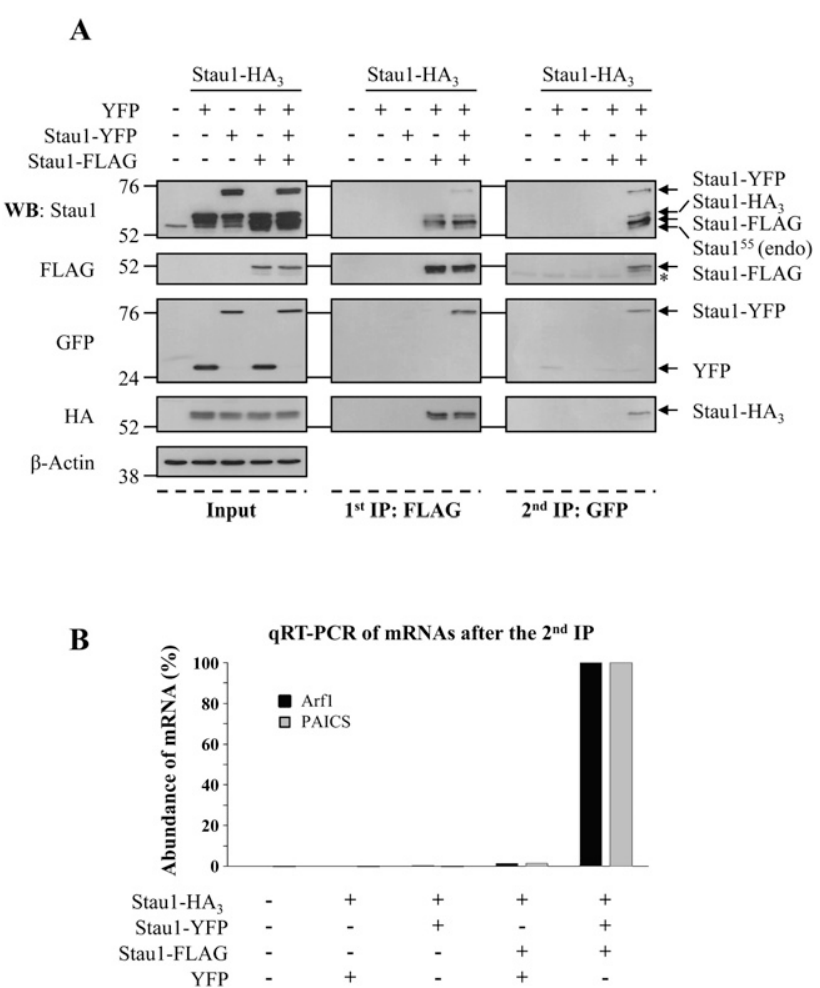

FIGURE 8. Staul forms RNA-containing higher-order complexes. (A) HEK293T cells were mock transfected or co-transfected with Staul-HA $\mathrm{H}_{3}$ expressor and either YFP, Stau1-YFP, or Stau1-Flag expressors as indicated. Proteins in the cell extracts were separated by SDS-PAGE (left panels, Input) or successively immunoprecipitated by first using anti-Flag antibody (middle panels, $1^{\text {st }}$ IP: FLAG) and then using anti-GFP antibody (right panels, $2^{\text {nd }}$ IP: GFP). Proteins were analyzed by Western blotting using anti-Staul, anti-Flag, antiGFP, and anti-HA antibodies, as indicated. Anti- $\beta$-actin antibody was used as a loading control. Molecular weight markers are indicated on the left side of the gels. (B) Immunoprecipitates obtained after the second round of immunoprecipitation were treated with TRIzol reagent to isolate putative Staul-bound RNAs. RNAs were qRTPCR amplified using oligonucleotide primers for ARF1 and PAICS mRNAs, two known Staul-bound mRNAs (Kim et al. 2005). This figure is representative of three independent experiments. 
This may not be crucial in the presence of functional dsRBD5 (Fig. 4C,D, Staul-Staul ${ }^{\Delta 2}$, Staul $1^{\Delta 2}-S_{\text {Staul }}{ }^{\Delta 2}$ ), but it may explain why Stau1 $1^{\Delta 2}$ and $S t a u 1^{\Delta 5}$ only poorly interact in live cells (Fig. 4D) when dsRBD5-mediated protein-protein interactions are prevented.

\section{Stau1 and the organization of mRNP complexes}

The involvement of both Staul RNA-binding activity and protein-protein interaction for Staul self-multimerization suggests that Staul may act as an organizer of mRNP formation. In this model, Staul recognizes and binds specific mRNAs to be transported via its RNA-binding domains and organizes these mRNAs into functional mRNP complexes through protein-protein interaction involving dsRBD5 and likely dsRBD2. Interestingly, two of the known Staul RNA substrates are known to form homodimers: bicoid mRNAs in Drosophila (Ferrandon et al. 1997) and HIV-1 genomic RNA in humans (Darlix et al. 1990). Therefore, complexes may be formed through both Staul and RNA self-association. This novel function of Staul in complex formation would be complementary to other known downstream functions of Staul in mRNA transport, translation, and decay. Moreover, Staul was shown to make direct protein-protein interaction with tubulin (Wickham et al. 1999), ribosomes (Luo et al. 2002; Brendel et al. 2004), the nonsense mediated factor Upf1 (Kim et al. 2005; Gong et al. 2009), and protein phosphatase I (Monshausen et al. 2002), proteins known to be present in Staul-containing mRNPs. Therefore, not only Staul can organize mRNPs through self-association, it can also attract additional RNP-associated proteins through direct interaction. Interestingly, all these interactions were mapped within the Stau1 TBD domain, a region that is not involved in RNA binding or Staul self-association. Therefore, Staul has the capability to select mRNAs, organize multimeric mRNA-protein structures, and attract additional proteins to form functional mRNPs.

Several data in the literature are consistent with this model. First, Stau1 is known to co-localize with mRNAs in mRNP complexes (Kiebler et al. 1999; Duchaine et al. 2000; Monshausen et al. 2001; Mallardo et al. 2003; Kanai et al. 2004; Furic et al. 2008) and directly bind mRNAs through a specific cis-acting element (Wickham et al. 1999; Kim et al. 2007), indicating that Staul has the capability to select mRNAs to be transported and form functional mRNPs. Second, biochemical and proteomic studies have shown that Staul is a component of large complexes that contain mRNA and protein cofactors (Krichevsky and Kosik 2001; Mallardo et al. 2003; Brendel et al. 2004; Villace et al. 2004). Its capacity to bind both mRNAs and specific mRNPassociated proteins via protein-protein interaction (see above) will facilitate the coordination between the fate of Stau1-bound mRNAs and proteins required to fulfill the functions. Third, relatively few Staul molecules were detected as monomers or dimers (Mallardo et al. 2003), indicating that Staul forms higher-order structures. The biochemical and BRET/PCA results (Figs. 6-8) that show the ability of Stau1 to form oligomers in live cells support this conclusion. Fourth, in situ hybridization using Stau1bound mRNA sequences as probes revealed that, as a consequence of Staul down-regulation, the amount of granules containing these specific mRNAs is reduced in dendrites (Kanai et al. 2004; Vessey et al. 2008). Although these data can be interpreted as an indication that Staul downregulation impairs mRNA transport, they may also indicate that Stau1 down-regulation also impairs mRNPs formation. Finally, the oligomeric nature of Staul-containing particles in dendrites was exemplified in mouse with a loss-of-function allele for Staul (Staul ${ }^{\text {tmlApa }}$ ). Whereas a transport-defective $\Delta$ Staul-YFP was not properly transported in dendrites following transfection in hippocampal neurons derived from Stau $1^{\text {tmlApa }}$ mouse, it was normally transported in neurons from wild-type mouse (Vessey et al. 2008). This result indicates that wild-type Staul can rescue the transport of $\Delta$ Staul, likely through an interaction that forms functional mRNPs to be transported in dendrites. A similar mechanism can be used to control the functions of mRNP complexes. Of particular interest is Staul $1^{\mathrm{i}}$, a Staul isoform that contains an insertion of six amino acids in dsRBD3 that impairs its ability to bind RNA and causes the formation of RNAbinding-defective particles (Duchaine et al. 2000; Monshausen et al. 2001). Its association with Staul in mRNPs modulates the RNA content of Staul-containing mRNPs and their functions.

In conclusion, our results suggest that the formation of large Stau1-containing mRNP complexes may be initiated by Staul itself through both RNA-binding and homophilic and heterophilic protein-protein interactions. This mechanism may represent a first step for the regulation of downstream Staul functions such as mRNA transport and localization (Kanai et al. 2004), nuclear transit (Martel et al. 2006), translation regulation (Dugre-Brisson et al. 2005), interaction with the cytoskeleton (Wickham et al. 1999), and Stau1mediated mRNA decay (Kim et al. 2005, 2007). Using sensitive and novel approaches, we were able for the first time to show that Staul dimers and most likely oligomers are associated with mRNAs. These techniques will contribute to our understanding of mRNA particle formation and transport and of how their misregulation may impair Stau1mediated cellular functions.

\section{MATERIALS AND METHODS}

\section{Antibodies and reagents}

Goat polyclonal and mouse monoclonal anti-GFP antibodies were obtained from Rockland and Roche Applied Science, respectively. Mouse monoclonal anti- $\beta$-actin clone AC-74 and mouse monoclonal anti-Flag M2 were obtained from Sigma-Aldrich. The mouse monoclonal anti-Stau1 (clone 11C6) and anti-HA (clone 
12CA5) antibodies were previously described (Dugre-Brisson et al. 2005). Anti-mouse and anti-goat polyclonal antibodies conjugated to horseradish peroxidase were obtained from DakoCytomation. $\mathrm{X}$-gal and imidazole were purchased from Bioshop. Western blot signals were detected using Western Lightning Chemiluminescence Reagent Plus (PerkinElmer Life Sciences).

\section{cDNA construction}

YFP-fused Stau1 mutants (Luo et al. 2002), Stau1-Rluc (ChatelChaix et al. 2004), Stau1-HA , Staul $^{\Delta 2}$-his $_{6}$ plasmids (Wickham et al. 1999), $\mathrm{GABA}_{\mathrm{B}} \mathrm{R} 1-\mathrm{YFP}, \mathrm{GABA}_{\mathrm{B}} \mathrm{R} 2-$ Rluc (Perroy et al. 2003), and PKAreg-Rluc ${ }^{\mathrm{F} 1}$ and PKAcatRluc ${ }^{\mathrm{F} 2}$ (Stefan et al. 2007) were previously described. Staul ${ }^{\Delta 2}$-Rluc and $S t a u 1^{\Delta 5}$-Rluc were constructed from Stau1-Rluc by the all-around technique. Oligonucleotides used to generate these constructs are listed in Table 1 . The $4 \mathrm{~K}$ mutant was constructed from the $2 \mathrm{~K}$ mutant (Dugre-Brisson et al. 2005 ) by the all-around technique (Table 1). The PCR fragment corresponding to PABP was digested with BamHI and cloned in pCMV-YFP-topaz (Packard Bioscience/PerkinElmer LifeSciences).

PCR fragments corresponding to dsRBD2, dsRBD3, dsRBD4, and dsRBD5 of Stau1 (Table 1) were digested with EcoRI and XhoI enzymes and cloned into pLexA pre-digested with EcoRI and XhoI. The PCR fragment corresponding to the TBD domain was digested with MunI and XhoI and cloned into pLexA pre- digested with EcoRI and XhoI. Full-length Stau1 was obtained by PCR amplification (Table 1) and digested with XhoI. This fragment was cloned into $\mathrm{pB} 42 \mathrm{AD}$ pre-digested with XhoI.

\section{Recombinant protein production and purification}

Bacterially expressed his $_{6}$-Staul and neutral endopeptidase his $_{6}$ NEP were purified as previously described (Wickham et al. 1999). Aliquots of the purified proteins were stored at $-80^{\circ} \mathrm{C}$ until use. Protein concentration was determined by the Bio-Rad dye reagent and BSA as a standard. For the pull-down assays, his ${ }_{6}$-Staul and his $_{6}$-NEP (as control) were immobilized on a Ni-NTA-agarose matrix and in vitro translated ${ }^{35}$ S-labeled Staul was loaded onto the column. After extensive washings, bound proteins were eluted with $300 \mathrm{mM}$ imidazole, and three successive elution fractions were analyzed by SDS-PAGE and autoradiography. In parallel, the in vitro translated ${ }^{35} \mathrm{~S}$-Staul extract was treated with $20 \mathrm{pg} / \mathrm{mL}$ RNase A for $1 \mathrm{~h}$ prior to loading.

\section{Yeast two-hybrid assay}

The EGY48 strain was transformed with the pLexA or pB42 plasmids containing the Staul fragments of interest. Yeast cells were grown in either synthetic growth media lacking the nutrients indicated or rich media.

TABLE 1. Primers used for PCR amplification

\begin{tabular}{|c|c|c|c|}
\hline Domain & Orientation & Restriction & Sequence \\
\hline Stau1 ${ }^{\Delta 2}$-Rluc & $\begin{array}{l}\text { Sense } \\
\text { Antisense }\end{array}$ & - & $\begin{array}{l}\text { 5'-GAGGTGAATGGAAGAGAATCCGAA-3' } \\
\text { 5'-CATAAGCTTGATATCCTGCAGACG-3' }\end{array}$ \\
\hline Stau $1^{\Delta 5}$-Rluc & $\begin{array}{l}\text { Sense } \\
\text { Antisense }\end{array}$ & - & $\begin{array}{l}\text { 5'-ATCCCACCGGCTAGAGCCACC-3' } \\
\text { 5'-CTCAGAGGGTCTCGTGAGAGG-3' }\end{array}$ \\
\hline Stau1 ${ }^{4 K_{-}}$Rluc & $\begin{array}{l}\text { Sense } \\
\text { Antisense }\end{array}$ & - & $\begin{array}{l}\text { 5'-GCACCAACGCGGCGGTGGCCA-3' } \\
\text { 5'-TGGCCACCGCCGCGTTGGTGC-3' }\end{array}$ \\
\hline Stau1-Rluc ${ }^{\mathrm{F} 1 / \mathrm{F} 2}$ & $\begin{array}{l}\text { Sense } \\
\text { Antisense }\end{array}$ & $\begin{array}{l}\text { Notl } \\
\text { Clal }\end{array}$ & $\begin{array}{l}\text { 5'-TAGCATGCGGCCGCATGAAACTTGGAAAAAAACC-3' } \\
\text { 5' - AGAGATATCGATGCACCTCCCACACACA -3' }\end{array}$ \\
\hline PABP-YFP & $\begin{array}{l}\text { Sense } \\
\text { Antisense }\end{array}$ & $\begin{array}{l}\text { BamHI } \\
\text { BamHI }\end{array}$ & $\begin{array}{l}\text { 5'-ACAAGGATCCGCCACCATGAACCCCAGTGCCC-3' } \\
\text { 5'-TTGTGGATCCGCAACAGTTGGAACACCGGT-3' }\end{array}$ \\
\hline dsRBD2-pLexA & $\begin{array}{l}\text { Sense } \\
\text { Antisense }\end{array}$ & $\begin{array}{c}\text { EcoRI } \\
\text { Xhol }\end{array}$ & $\begin{array}{l}\text { 5'-TAGATATCGAATTCGCCGCCATGGGATACCCATTTCCAG-3' } \\
\text { 5'-TATACTCGAGGATATCCAGGATCCTCAA-3' }\end{array}$ \\
\hline dsRBD3-pLexA & $\begin{array}{l}\text { Sense } \\
\text { Antisense }\end{array}$ & $\begin{array}{l}\text { EcoRI } \\
\text { Xhol }\end{array}$ & $\begin{array}{l}\text { 5'-TAGATATCGAATTCGCCGCCATGGGAAAATCTGAAATAAGT-3' } \\
\text { 5'-TATAACTCGAGGATATCAACTGCAGTGCAGG-3' }\end{array}$ \\
\hline dsRBD4-pLexA & $\begin{array}{l}\text { Sense } \\
\text { Antisense }\end{array}$ & $\begin{array}{l}\text { EcoRI } \\
\text { Xhol }\end{array}$ & $\begin{array}{l}\text { 5'-TAGATATCGAATTCGCCGCCATGGGAAATCCGATTAGC-3' } \\
\text { 5'-TATACTCGAGGATATCAAGGATCTCCAG-3' }\end{array}$ \\
\hline dsRBD5-pLexA & $\begin{array}{l}\text { Sense } \\
\text { Antisense }\end{array}$ & $\begin{array}{l}\text { EcoRI } \\
\text { Xhol }\end{array}$ & $\begin{array}{l}\text { 5'-TAGATATCGAATTCGCCGCCATGGGACCCTCTGAGCAA-3' } \\
\text { 5'-TATACTCGAGGATATCGCACCTCCCACACAC-3' }\end{array}$ \\
\hline TBD-pLexA & $\begin{array}{l}\text { Sense } \\
\text { Antisense }\end{array}$ & $\begin{array}{l}\text { Munl } \\
\text { Xhol }\end{array}$ & $\begin{array}{l}\text { 5'-TAGATATCCAATTGGCCGCCATGGGAAAACCCGCACT-3' } \\
\text { 5'-TATACTCGAGGATATCGGCAGTTACCGTGGC-3' }\end{array}$ \\
\hline Stau1-pB42AD & $\begin{array}{l}\text { Sense } \\
\text { Antisense }\end{array}$ & $\begin{array}{l}\text { Xhol } \\
\text { Xhol }\end{array}$ & $\begin{array}{l}\text { 5'-GATGCTCGAGATGAAACTTGGAAAAAAACC-3' } \\
\text { 5'-TATACTCGAGGATATCGCACCTCCCACACAC-3' }\end{array}$ \\
\hline Arf1 & $\begin{array}{l}\text { Sense } \\
\text { Antisense }\end{array}$ & - & $\begin{array}{l}\text { 5'-TCAACGTGGAAACCGTGGAGTACA-3' } \\
\text { 5'-AGATCAGGCCTTGTGTGTTCTGGA-3' }\end{array}$ \\
\hline PAICS & $\begin{array}{l}\text { Sense } \\
\text { Antisense }\end{array}$ & - & $\begin{array}{l}\text { 5'-TTTGGGACCAGTGATGTCTGGGAA-3' } \\
\text { 5'-TTGAACAGCCAAGACCACTGGGTA-3' }\end{array}$ \\
\hline
\end{tabular}

Restriction sites are underlined and shown in boldface. 


\section{Cell culture, transfection, and BRET assay}

Human embryonic kidney (HEK) 293T cells were cultured in Dulbecco's Modified Eagle Medium (Invitrogen) supplemented with $10 \%$ cosmic calf serum (HyClone). Transfections were carried out with the Lipofectamine 2000 reagent (Invitrogen). BRET experiments were carried out as described (Chatel-Chaix et al. 2004, 2007). Briefly, cells were plated into 12-well plates at 120,000 cells per well. Twenty-four hours later, cells were transfected with constant amounts of plasmids coding for Rluc-tagged fusion protein $(10 \mathrm{ng})$ and increasing amounts of plasmids coding for YFP-tagged proteins (0-500 ng) as indicated. BRET assays were performed $24 \mathrm{~h}$ post-transfection in live cells. Cells were washed twice with phosphate buffered saline (PBS) at room temperature and diluted to $10^{6}$ cells per $\mathrm{mL}$. Coelenterazine $\mathrm{H}$ (NanoLight Technology) was added to $90 \mu \mathrm{L}$ of cells at a final concentration of $5 \mu \mathrm{M}$. A saturation curve can be drawn when the Rluc-tagged protein becomes saturated by YFP-tagged proteins, and an optimal BRET ratio can be calculated at saturation for a specific proteinprotein interaction. Luminescence $(440-500-\mathrm{nm})$ as well as total and transmitted fluorescence (510-590-nm) emissions were measured using a Fusion $\alpha$-FP apparatus (PerkinElmer). The BRET ratio was defined as [(emission at 510 to $590 \mathrm{~nm})-($ emission at 440 to $500 \mathrm{~nm}) \times \mathrm{Cf}] /($ emission at $440-500 \mathrm{~nm})$, where Cf corresponds to (emission at 510 to $590 \mathrm{~nm}) /($ emission at 440 to $500 \mathrm{~nm}$ ) when Rlucfused protein is expressed alone. The total YFP activity/Rluc activity ratio reflects the relative levels of activity of the two fusion proteins in the cells. When BRET was carried out in cell extracts, cells were lysed in $200 \mu \mathrm{L}$ of lysis buffer $(0.1 \mathrm{M}$ Tris-Cl at $\mathrm{pH} 7.9,0.5 \%$ Nonidet P40, $1 \mathrm{mM}$ DTT) and incubated for $15 \mathrm{~min}$ at room temperature with or without $30 \mu \mathrm{g} / \mathrm{mL}$ of RNase A (Fermentas).

For BRET between Staul-Rluc and RNA, $1 \mu \mathrm{L}$ of SYTO 14 (Invitrogen), an RNA selective fluorescence dye used as energy acceptor, was added to 100,000 cells or to cell extracts and was incubated for $20 \mathrm{~min}$ at room temperature. Because the emission of SYTO14 is shifted to the red compared with that of YFP, a 550 long-pass filter (Chroma) was used as acceptor filter. The BRET ratio was calculated as described before by making the ratio of the acceptor filter over the donor filter.

\section{Microscopy}

COS-7 cells were transfected with plasmids (500 ng) coding for different Staul mutants using PEI as previously described (Guerra-Crespo et al. 2003). Cells were fixed in a freshly made $4 \%$ paraformaldehyde solution in PBS for $20 \mathrm{~min}$ at room temperature and permeabilized with $0.1 \%$ Triton X-100 in PBS. Coverslips were mounted on microscope slides using DAKO fluorescent medium and images were taken on a Nikon TE2000U equipped with a CoolSnap fx CCD camera, a $60 \times$ objective (Pan Apo, N.A. 1.4), and a YFP filter cube (Chroma \#41028). Images were treated in Image $1.43 \mathrm{~d}$. The YFP channels were gamma corrected (0.5), the black level was subtracted (modal value), and the brightness and contrast were set to $0.5 \%$ saturation.

\section{Immunoprecipitation and qRT-PCR}

HEK293T cells transfected with the indicated plasmids were harvested 24 h post-transfection, washed three times in ice-cold PBS, and lysed in buffer $(50 \mathrm{mM}$ Tris-Cl at $\mathrm{pH} 7.5,1 \%$ Triton X-100, 15 mM EGTA, $150 \mathrm{mM} \mathrm{NaCl}, 1 \mathrm{mM}$ DTT, Complete EDTA-free protease inhibitor cocktail [Roche Applied Science]) supplemented or not with RNaseOUT RNase inhibitor (Invitrogen). Lysates were cleared by centrifugation at $17,000 \mathrm{~g}$ for $15 \mathrm{~min}$. The supernatant was incubated with or without $30 \mu \mathrm{g} / \mathrm{mL}$ RNase A for $10 \mathrm{~min}$ at $37^{\circ} \mathrm{C}$ and then pre-cleared with protein G-sepharose (GE Healthcare) for $45 \mathrm{~min}$ at $4^{\circ} \mathrm{C}$. After centrifugation, the supernatant was incubated with goat polyclonal anti-GFP antibodies for $2 \mathrm{~h}$ at $4^{\circ} \mathrm{C}$ and then with protein $\mathrm{G}$-sepharose for $90 \mathrm{~min}$ at $4^{\circ} \mathrm{C}$. Immune complexes were washed three times with the lysis buffer and eluted from the resin by heating for $5 \mathrm{~min}$ at $95^{\circ} \mathrm{C}$ in $2 \times$ protein loading buffer (125 mM Tris-Cl at pH 6.8, 10\% glycerol, 3.3\% SDS, 0.2 M DTT, and $0.04 \%$ bromophenol blue). Proteins were analyzed by SDSPAGE and Western blotting using monoclonal antibodies against the indicated proteins.

For the successive immunoprecipitation experiments, lysates were prepared as described above except that DTT and RNase inhibitor were omitted. The first immunoprecipitation was carried out with anti-Flag M2 affinity gel (Sigma-Aldrich) and eluted with the Flag peptide (Sigma-Aldrich) as described previously (ChatelChaix et al. 2008). The eluate was then pre-cleared with protein G-sepharose and the second immunoprecipitation was performed with polyclonal anti-GFP as described above. One-third of the immune complex was analyzed by SDS-PAGE and Western blotting and two-thirds was used for qRT-PRC analysis. To this end, RNA was isolated from the immunoprecipitates using the TRIzol Reagent (Invitrogen) according to the manufacturer's procedure. RNA was resuspended in $50 \mu \mathrm{L}$ of water and digested with DNase using the TURBO DNA-free kit (Ambion). Reverse transcription reactions were done with $4 \mu \mathrm{L}$ of RNA using the MuLV RT enzyme from the GeneAmp RNA PCR kit (Applied Biosystems), according to the manufacturer's procedure. Specific antisense primers used to produce the cDNAs were 5'-AGATCAGGCCTTGTGTGTTCTGGA-3' for Arf1 and 5' -TTGAACAGCCAAGACCACTGGGTA-3' for PAICS. Resulting cDNAs were qPCR amplified using the LightCycler 480 SYBR Green I Master kit (Roche) and the LightCycler 480 instrument (Roche). Sense and antisense primers are described in Table 1.

\section{ACKNOWLEDGMENTS}

We thank Francis Goyette, Phu Vinh On, Monique Vasseur, Louise Cournoyer, and Linda Huang for technical assistance. C.M., T.D., V.T., and S.D.-B. were supported by studentships from the Natural Sciences and Engineering Research Council of Canada (NSERC), and K.B. received studentships from the Fonds de Recherche en Santé du Québec. This work was supported by a grant from NSERC to L.D.G.

Received March 30, 2009; accepted November 19, 2009.

\section{REFERENCES}

Brendel C, Rehbein M, Kreienkamp HJ, Buck F, Richter D, Kindler S. 2004. Characterization of Staufen 1 ribonucleoprotein complexes. Biochem J 384: 239-246.

Buchner G, Bassi MT, Andolfi G, Ballabio A, Franco B. 1999. Identification of a novel homolog of the Drosophila staufen protein in the chromosome 8q13-q21.1 region. Genomics 62: $113-118$.

Bycroft M, Grunert S, Murzin AG, Proctor M, St Johnston D. 1995. NMR solution structure of a dsRNA binding domain from 
Drosophila staufen protein reveals homology to the N-terminal domain of ribosomal protein S5. EMBO J 14: 3563-3571.

Chatel-Chaix L, Clement JF, Martel C, Beriault V, Gatignol A, DesGroseillers L, Mouland AJ. 2004. Identification of Staufen in the human immunodeficiency virus type $1 \mathrm{Gag}$ ribonucleoprotein complex and a role in generating infectious viral particles. Mol Cell Biol 24: 2637-2648.

Chatel-Chaix L, Abrahamyan L, Frechina C, Mouland AJ, DesGroseillers L. 2007. The host protein Staufen1 participates in HIV-1 assembly in live cells by influencing pr55Gag multimerization. J Virol 81: 6216-6230.

Chatel-Chaix L, Boulay K, Mouland AJ, DesGroseillers L. 2008. The host protein Staufen1 interacts with the Pr55Gag zinc fingers and regulates HIV-1 assembly via its N-terminus. Retrovirology 5: 41. doi: 10.1186/1742-4690-5-41.

Daher A, Longuet M, Dorin D, Bois F, Segeral E, Bannwarth S, Battisti PL, Purcell DF, Benarous R, Vaquero C, et al. 2001. Two dimerization domains in the trans-activation response RNAbinding protein (TRBP) individually reverse the protein kinase $\mathrm{R}$ inhibition of HIV-1 long terminal repeat expression. J Biol Chem 276: 33899-33905.

Darlix JL, Gabus C, Nugeyre MT, Clavel F, Barre-Sinoussi F. 1990. Cis elements and trans-acting factors involved in the RNA dimerization of the human immunodeficiency virus HIV-1. J Mol Biol 216: 689-699.

DesGroseillers L, Lemieux N. 1996. Localization of a human doublestranded RNA-binding protein gene (STAU) to band 20q13.1 by fluorescence in situ hybridization. Genomics 36: 527-529.

Dubnau J, Chiang AS, Grady L, Barditch J, Gossweiler S, McNeil J, Smith P, Buldoc F, Scott R, Certa U, et al. 2003. The Staufen/ pumilio pathway is involved in Drosophila long-term memory. Curr Biol 13: 286-296.

Duchaine T, Wang HJ, Luo M, Steinberg SV, Nabi IR, DesGroseillers L. 2000. A novel murine Staufen isoform modulates the RNA content of Staufen complexes. Mol Cell Biol 20: 5592-5601.

Duchaine TF, Hemraj I, Furic L, Deitinghoff A, Kiebler MA, DesGroseillers L. 2002. Staufen2 isoforms localize to the somatodendritic domain of neurons and interact with different organelles. J Cell Sci 115: 3285-3295.

Dugre-Brisson S, Elvira G, Boulay K, Chatel-Chaix L, Mouland AJ, DesGroseillers L. 2005. Interaction of Staufen1 with the $5^{\prime}$ end of mRNA facilitates translation of these RNAs. Nucleic Acids Res 33: 4797-4812.

Ferrandon D, Koch I, Westhof E, Nusslein-Volhard C. 1997. RNARNA interaction is required for the formation of specific bicoid mRNA 3' UTR-STAUFEN ribonucleoprotein particles. EMBO J 16: $1751-1758$.

Furic L, Maher-Laporte M, DesGroseillers L. 2008. A genome-wide approach identifies distinct but overlapping subsets of cellular mRNAs associated with Staufen1- and Staufen2-containing ribonucleoprotein complexes. RNA 14: 324-335.

Gong C, Kim YK, Woeller CF, Tang Y, Maquat LE. 2009. SMD and NMD are competitive pathways that contribute to myogenesis: Effects on PAX3 and myogenin mRNAs. Genes \& Dev 23: 5466.

Guerra-Crespo M, Charli JL, Rosales-Garcia VH, Pedraza-Alva G, Perez-Martinez L. 2003. Polyethylenimine improves the transfection efficiency of primary cultures of post-mitotic rat fetal hypothalamic neurons. J Neurosci Methods 127: 179-192.

Hamdan FF, Percherancier Y, Breton B, Bouvier M. 2006. Monitoring protein-protein interactions in living cells by bioluminescence resonance energy transfer (BRET). In Current protocols in neuroscience (ed. D Sibley), Chap. 5, Unit 5.23. Wiley, New York.

Hitti EG, Sallacz NB, Schoft VK, Jantsch MF. 2004. Oligomerization activity of a double-stranded RNA-binding domain. FEBS Lett 574: 25-30.

Kanai Y, Dohmae N, Hirokawa N. 2004. Kinesin transports RNA: Isolation and characterization of an RNA-transporting granule. Neuron 43: 513-525.
Kharrat A, Macias MJ, Gibson TJ, Nilges M, Pastore A. 1995. Structure of the dsRNA binding domain of E. coli RNase III. EMBO J 14: 3572-3584.

Kiebler MA, Hemraj I, Verkade P, Kohrmann M, Fortes P, Marion RM, Ortin J, Dotti CG. 1999. The mammalian staufen protein localizes to the somatodendritic domain of cultured hippocampal neurons: Implications for its involvement in mRNA transport. J Neurosci 19: 288-297.

Kim YK, Furic L, DesGroseillers L, Maquat LE. 2005. Mammalian Staufen1 recruits Upf1 to specific mRNA 3'UTRs so as to elicit mRNA decay. Cell 120: 195-208.

Kim YK, Furic L, Parisien M, Major F, DesGroseillers L, Maquat LE. 2007. Staufen 1 regulates diverse classes of mammalian transcripts. EMBO J 26: 2670-2681.

Kim-Ha J, Kerr K, Macdonald PM. 1995. Translational regulation of oskar mRNA by bruno, an ovarian RNA-binding protein, is essential. Cell 81: 403-412.

Krichevsky AM, Kosik KS. 2001. Neuronal RNA granules: A link between RNA localization and stimulation-dependent translation. Neuron 32: 683-696.

Lamontagne B, Tremblay A, Abou Elela S. 2000. The N-terminal domain that distinguishes yeast from bacterial RNase III contains a dimerization signal required for efficient double-stranded RNA cleavage. Mol Cell Biol 20: 1104-1115.

Lebeau G, Maher-Laporte M, Topolnik L, Laurent CE, Sossin W, DesGroseillers L, Lacaille JC. 2008. Staufen1 regulation of protein synthesis-dependent long-term potentiation and synaptic function in hippocampal pyramidal cells. Mol Cell Biol 28: 2896-2907.

Lemaire PA, Lary J, Cole JL. 2005. Mechanism of PKR activation: Dimerization and kinase activation in the absence of doublestranded RNA. J Mol Biol 345: 81-90.

Liu J, Hu JY, Wu F, Schwartz JH, Schacher S. 2006. Two mRNAbinding proteins regulate the distribution of syntaxin mRNA in Aplysia sensory neurons. J Neurosci 26: 5204-5214.

Lunde BM, Moore C, Varani G. 2007. RNA-binding proteins: Modular design for efficient function. Nat Rev Mol Cell Biol 8: 479-490.

Luo M, Duchaine TF, DesGroseillers L. 2002. Molecular mapping of the determinants involved in human Staufen-ribosome association. Biochem J 365: 817-824.

Mallardo M, Deitinghoff A, Muller J, Goetze B, Macchi P, Peters C, Kiebler MA. 2003. Isolation and characterization of Staufencontaining ribonucleoprotein particles from rat brain. Proc Natl Acad Sci 100: 2100-2105.

Marianayagam NJ, Sunde M, Matthews JM. 2004. The power of two: Protein dimerization in biology. Trends Biochem Sci 29: 618625.

Marion RM, Fortes P, Beloso A, Dotti C, Ortin J. 1999. A human sequence homologue of Staufen is an RNA-binding protein that is associated with polysomes and localizes to the rough endoplasmic reticulum. Mol Cell Biol 19: 2212-2219.

Martel C, Macchi P, Furic L, Kiebler MA, DesGroseillers L. 2006. Staufen 1 is imported into the nucleolus via a bipartite nuclear localization signal and several modulatory determinants. Biochem J 393: 245-254.

Martin KC, Ephrussi A. 2009. mRNA localization: Gene expression in the spatial dimension. Cell 136: 719-730.

Micklem DR, Adams J, Grunert S, St Johnston D. 2000. Distinct roles of two conserved Staufen domains in oskar mRNA localization and translation. EMBO J 19: 1366-1377.

Monshausen M, Putz U, Rehbein M, Schweizer M, DesGroseillers L, Kuhl D, Richter D, Kindler S. 2001. Two rat brain staufen isoforms differentially bind RNA. J Neurochem 76: 155-165.

Monshausen M, Rehbein M, Richter D, Kindler S. 2002. The RNAbinding protein Staufen from rat brain interacts with protein phosphatase-1. J Neurochem 81: 557-564.

Nanduri S, Rahman F, Williams BR, Qin J. 2000. A dynamically tuned double-stranded RNA binding mechanism for the activation of antiviral kinase PKR. EMBO J 19: 5567-5574. 
Perroy J, Adam L, Qanbar R, Chenier S, Bouvier M. 2003. Phosphorylation-independent desensitization of GABA(B) receptor by GRK4. EMBO J 22: 3816-3824.

Peters GA, Hartmann R, Qin J, Sen GC. 2001. Modular structure of PACT: Distinct domains for binding and activating PKR. Mol Cell Biol 21: 1908-1920.

Ramos A, Grunert S, Adams J, Micklem DR, Proctor MR, Freund S, Bycroft M, St Johnston D, Varani G. 2000. RNA recognition by a Staufen double-stranded RNA-binding domain. EMBO J 19: 997-1009.

Rodriguez AJ, Czaplinski K, Condeelis JS, Singer RH. 2008. Mechanisms and cellular roles of local protein synthesis in mammalian cells. Curr Opin Cell Biol 20: 144-149.

Ryter JM, Schultz SC. 1998. Molecular basis of double-stranded RNAprotein interactions: Structure of a dsRNA-binding domain complexed with dsRNA. EMBO J 17: 7505-7513.

Sossin WS, DesGroseillers L. 2006. Intracellular trafficking of RNA in neurons. Traffic 7: 1581-1589.

St Johnston D, Beuchle D, Nusslein-Volhard C. 1991. Staufen, a gene required to localize maternal RNAs in the Drosophila egg. Cell 66: $51-63$.
Stefan E, Aquin S, Berger N, Landry CR, Nyfeler B, Bouvier M, Michnick SW. 2007. Quantification of dynamic protein complexes using Renilla luciferase fragment complementation applied to protein kinase A activities in vivo. Proc Natl Acad Sci 104: 16916-16921.

Valente L, Nishikura K. 2007. RNA binding-independent dimerization of adenosine deaminases acting on RNA and dominant negative effects of nonfunctional subunits on dimer functions. J Biol Chem 282: 16054-16061.

Vessey JP, Macchi P, Stein JM, Mikl M, Hawker KN, Vogelsang P, Wieczorek K, Vendra G, Riefler J, Tubing F, et al. 2008. A loss of function allele for murine Staufen1 leads to impairment of dendritic Staufen1-RNP delivery and dendritic spine morphogenesis. Proc Natl Acad Sci 105: 16374-16379.

Villace P, Marion RM, Ortin J. 2004. The composition of Staufencontaining RNA granules from human cells indicates their role in the regulated transport and translation of messenger RNAs. Nucleic Acids Res 32: 2411-2420.

Wickham L, Duchaine T, Luo M, Nabi IR, DesGroseillers L. 1999. Mammalian staufen is a double-stranded-RNA- and tubulinbinding protein which localizes to the rough endoplasmic reticulum. Mol Cell Biol 19: 2220-2230. 

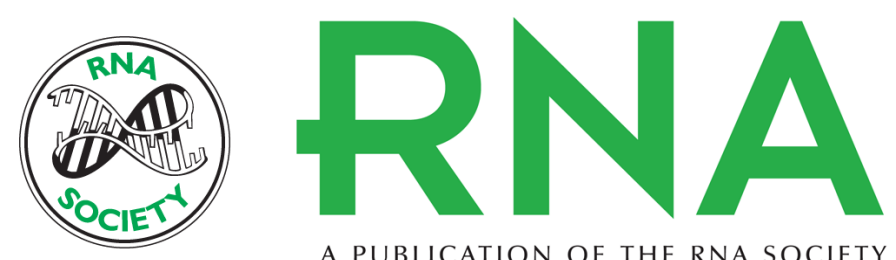

A PUBLICATION OF THE RNA SOCIETY

\section{Multimerization of Staufen1 in live cells}

Catherine Martel, Samuel Dugré-Brisson, Karine Boulay, et al.

RNA 2010 16: 585-597 originally published online January 14, 2010

Access the most recent version at doi:10.1261/rna.1664210

\section{References This article cites 52 articles, 24 of which can be accessed free at:} http://rnajournal.cshlp.org/content/16/3/585.full.html\#ref-list-1

\section{License}

Email Alerting Receive free email alerts when new articles cite this article - sign up in the box at the Service top right corner of the article or click here.

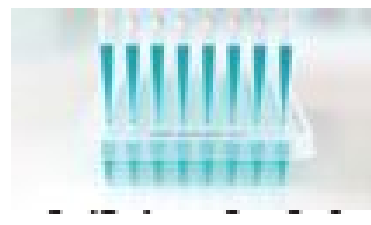

